\title{
EXPERIMENTAL AND NUMERICAL CURVED FLOW STUDY FOR METROLOGY PURPOSES
}

\author{
F. Sánchez, A. Gómez, M. Toledo, P. Quinto, \& V. Zurita. \\ Laboratorio de Ingeniería Térmica e Hidráulica Aplicada. \\ SEPI-ESIME-IPN-COFAA \\ U.P. "Adolfo López Mateos", edif. 5, 3er piso C.P. 07738, México D. F. \\ Tel. (5) 7296000 ext. 54754 E-mail: fsilva maya.esimez.ipn.mx; abdielgomez@hotmail.com
}

Received: September $22^{\text {th }} 2000$ and accepted May $8^{\text {th }} 2002$

\begin{abstract}
Most of the current volumetric flow measurement methods represent an important investment for many industries. However, when the accuracy requirements for the processes are not too high, curved pipes could be used as primary elements for this purpose. Unfortunately, there is a lack of information for this device as flowmeter, so more experimental and numerical research is required for its full characterization. This paper reports the experimental work conducted on three commercial $90^{\circ}$ elbows, installed horizontally. All of them were short curvature radius with $0.75,1$ and 1.5 inches of internal diameter; water maintained at $24{ }^{\circ} \mathrm{C}$ was employed for the experiments. Pressure taps on the elbows were located at $45 \circ$ along the curvature radius, on both inside and outside the wall. The performance of the elbow was compared against a calibrated orifice plate. A numerical study using CFD-2000 provided more information about the pressure and velocity fields, so it was posible to develop a model to describe the curvature effects on the radial pressure gradient. Experimental results show that there is a greater sensibility of the element when the curvature radius decreases, this means, a larger pressure gradient is obtained. If this radial pressure gradient is used in the typical equation for the orifice plate, a good concordance between the measurements of both elements is observed.
\end{abstract}

\section{RESUMEN}

La mayor parte de los métodos de medición de flujo volumétrico representan una inversión considerable para muchas industrias. Sin embargo, cuando los requerimientos de precisión en los procesos no son muy altos, los tubos curvados pueden ser utilizados como elementos primarios para este propósito. Desafortunadamente, hay mucha falta de información sobre este tipo de medidores de flujo, de manera que más investigación experimental y teórica se requiere para su completa caracterización. Este trabajo reporta el trabajo experimental conducido en tres codos comerciales a $90^{\circ}$ instalados horizontalmente, con radios de curvatura pequeños y un diámetro interno de $0.75,1$ y 1.5 pulgadas respectivamente y tomas de presión a $45^{\circ}$ sobre el radio de curvatura. Los resultados experimentales muestran una gran sensibilidad del elemento cuando el radio de curvatura disminuye, dando un mayor gradiente de presión, del cual se puede derivar el valor del flujo.

KEYWORDS: Flow measurement, two-phase flow, elbows.

\section{INTRODUCTION}

Flow measurement is very important for industries in handling fluids in their processes. The correct flow rate determination has many repercussions in the economy and life of an enterprise, because its market 
presence could be affected for the low quality of its products, as well as their selling and purchasing of fluids.

There is a great variety of flow measurement methods, using several physical principles involving high technologies like ultrasound and laser, but there are also instruments using well-known techniques like differential pressure drop (DP). These DP methods have a very important place among the flow measurement instruments, and a high percentage of the installed instrumentation belongs to this classification [10].

In the DP instruments, the flow rate is determined using the pressure drop provoked by an obstruction, which is normally inserted in the pipe. The flow equations for all the DP flow instruments remain the same, and are based on the Bernoulli's equation, corrected by some factors obtained from experimental observations taking into account the geometry of the primary element.

The obstruction could be of different configurations, for instance, the venturimeter, the orifice plate, Dall's pipe, etc. The conventional way of using these instruments is to insert them into an horizontal or vertical pipe with a normalized upward and downward length for the reduction of inlet and exit effects; the pipe must be of constant section [11].

As the flow rate is determined by using the pressure difference between two points along the pipe, theoretically it is possible to get it using any kind of device, the only condition is to have a pressure drop. We can use this same principle for elbows, which are fittings commonly used in industrial installations, mainly for changing the flow direction or flexibility purposes. These fittings provoke also a pressure drop due to their geometrical configuration..

The use of elbows as primary elements for fluid flow measuring is not in fact a new method, but its use has not been diffused due to the lack of studies for its full characterization. This method has many advantages like its simplicity, low cost compared against any other flow measurement device, simplicity of calibration and installation, and it does not introduce additional pressure drop, saving energy that, otherwise will be needed as additional power for the fluid transport, with the corresponding economical effect.

As in all the DP instruments, the flow rate is determined using the continuity equation $Q=V A, A$ is the cross section area of the flow pipe, $\mathrm{V}$ is the average fluid velocity in the cross section of the pipe, which is determined using the Bernoulli's equation: $V=\sqrt{2 \text { ? } \mathrm{P} / \rho}$. If we take as an example the orifice plate, the volumetric flow rate is given by the following equation:

$$
Q=\frac{\mathrm{C}_{\mathrm{P}} \mathrm{pd}^{2}}{4} \sqrt{\frac{2 ? \mathrm{P}}{?}}
$$

In the case of elbows used as DP elements, the fluid flow rate is also determined taking into account the pressure difference between two points. The main difference with the conventional DP instruments is that the differential pressure, in the former case, is radial instead of axial. If we apply the Euler equation to a current line, we obtain:

$$
\frac{1}{\rho} \frac{\partial P}{\partial r}=\frac{V^{2}}{r}
$$

Using this equation, we can get the average velocity in the elbow that is expressed as:

$$
V=\sqrt{\frac{? P_{r}}{\rho \ln \left(r_{e} / r_{b}\right)}}
$$


So that $\mathrm{V}$ is the average tangential velocity in the transversal plane of the elbow. Therefore, the volumetric flow in the elbow could be written as:

$$
Q=\frac{C_{c} p D^{2}}{4} \sqrt{\frac{{ }^{P} P_{r}}{\rho \ln \left(D / r_{b}+1\right)}}
$$

Coefficients CP and CC in Equations (1) and (4) take into account the friction between the fluid and the walls of the pipe and the flow irreversibilities as well. These equations are quite similar in shape, both have a pressure difference DP multiplied by parameters related to the element geometry, which could be taken as coefficients. Then, it is possible to use the radial pressure drop DPr in the elbow to determine the flow rate in the system. Of course, it is necessary to determine whether the DPr is big enough in order to increase the sensibility of the instrument.

The main purpose of this work is to demonstrate the viability of using the radial pressure gradient of the elbows for the volumetric flow determination and to determine the best pressure taps position. The experimental results were compared using a calibrated orifice plate.

\section{FLOW BEHAVIOR IN CURVED PIPES}

When a flow enters into a curved pipe of any type of cross section, there is an unbalance between the centrifugal force and the radial pressure gradient, acting in opposite directions. In order to maintain the momentum balance, the fluid particles in the high velocity zone (the center of the cross section) move toward the external wall pushing the particles of this zone to the center of the pipe and then to the internal wall. This effect produces a transversal fluid circulation; i.e. the fluid is moving from the internal to the external wall.

Boundary layer is developed first in the external wall and travels through the sides to the internal wall where both flows interact, giving origin to a flow, which leaves the internal wall and is directed toward the external one, forming a secondary flow [8]. The axial pressure gradient produces primary flow separation when Dean number $\left[\operatorname{Re}\left(\mathrm{D} / \mathrm{rb}_{\mathrm{b}}\right)^{1 / 2}\right]$ is too large. Curvature radius has also a great influence in the separation phenomena. The flow in curved pipes depends mainly of three parameters: Reynolds number, Dean number and curvature radius.

\section{NUMERICAL ANALYSIS}

The continuity and Navier-Stokes equations for the flow field determination in the curved pipe must be solved in the body fitted coordinate system shown in figure 1. These equations are:

$$
\begin{gathered}
\nabla \cdot(? V \quad)=0 \\
\frac{\mathrm{DV}}{\mathrm{Dt}}=\mathrm{g}-\frac{1}{\rho} \nabla \mathrm{P}+? \nabla^{2} \mathrm{~V}+\frac{?}{3} \nabla(\nabla \cdot \mathrm{V})
\end{gathered}
$$

In the case under study, the above equations are applied to an incompressible, steady state turbulent flow, with the boundary conditions shown in table I. The CFD-2000 code was used for their solution. 


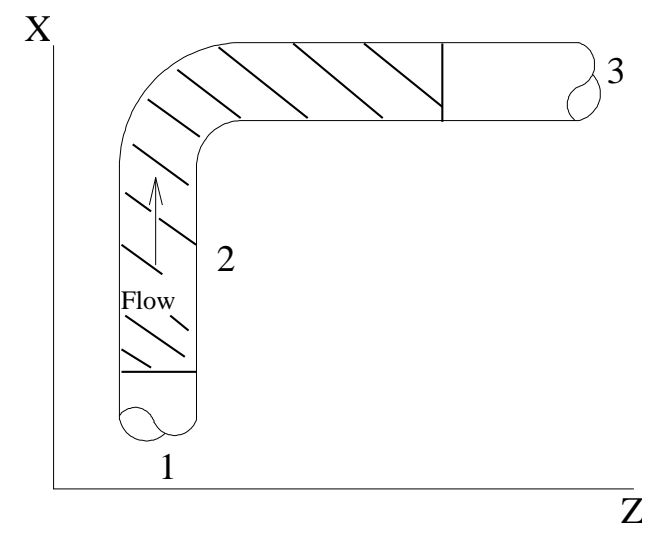

Figure 1. Control volume

Table I. Control volume boundary conditions

\begin{tabular}{|c|c|}
\hline Boundary & Condition \\
\hline 1 & $\begin{array}{l}\text { - Developed flow } \\
\text { - Constant profile } U_{x} \text { velocity } \\
\text { - Velocity } \bigcup_{y} \text { and } U_{z} \text { zero }\end{array}$ \\
\hline 2 & $\begin{array}{l}\text { - Developed flow } \\
\text { - Constant profile } U_{z} \text { velocity } \\
\text { - Velocity } U_{x} \text { and } U_{y} \text { zero }\end{array}$ \\
\hline 3 & $\begin{array}{l}\text { - Solid Wall } \\
\text { - No slip condition } \\
\text { - No temperature jump condition }\end{array}$ \\
\hline
\end{tabular}

The k-e turbulence model used in this study is already included in the code. In this model two differential equations whose independent variables are the turbulent kinetic energy $k$ and the turbulence energy dissipation rate $\varepsilon$ are solved. These variables are:

$$
\begin{gathered}
\kappa=\frac{1}{2} \overline{u_{i} u_{i}} \\
\varepsilon=\nu \frac{\overline{\partial u_{i} \partial u_{i}}}{\partial x_{k} \partial x_{k}}
\end{gathered}
$$

\section{NUMERICAL ANALYSIS USING CFD-2000 CODE}

CFD-2000 code was employed for the numerical analysis. The procedure was as follows: the volume control was established as in figure 2. It consists of a smooth zone with 30D length before the elbow and a 25D length after it, with a 44-mm internal diameter pipe, in order to avoid inlet and outlet effects. Three different curvature radius were used for the study: 20, 100 and $230 \mathrm{~mm}$, so curvature ratio d/R were 2.2, 0.44 and 0.191 respectively. Region 1 is located upstream of the elbow, region 2 corresponds to the location of the elbow, whereas region 3 is located downstream of the elbow. They all formed the control volume and each one was divided in a number of nodes indicated in table II. The flow characteristics for the experimental and numerical study are shown in table 3 . The Reynolds numbers here analyzed correspond to the zone of developed flow i.e. 30D before the elbow. 


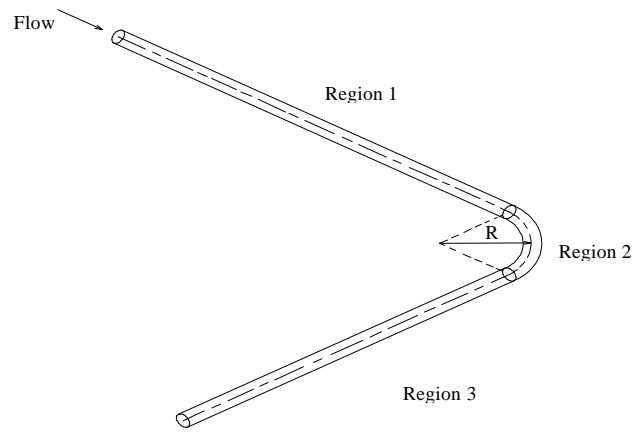

Figure 2. Control volume for the numerical analysis.

Table II. Nodal distribution of the geometry model

\begin{tabular}{|l|c|c|c|}
\hline & Region 1 & Region 2 & Region 3 \\
\hline I Direction & 10 & 16 & 10 \\
\hline J Direction & 10 & 10 & 10 \\
\hline K Direction & 10 & 10 & 10 \\
\hline
\end{tabular}

\section{EXPERIMENTAL STUDY}

The experimental facility constructed for this work is shown in figure 3 . Details on work station are presented in figure 4.

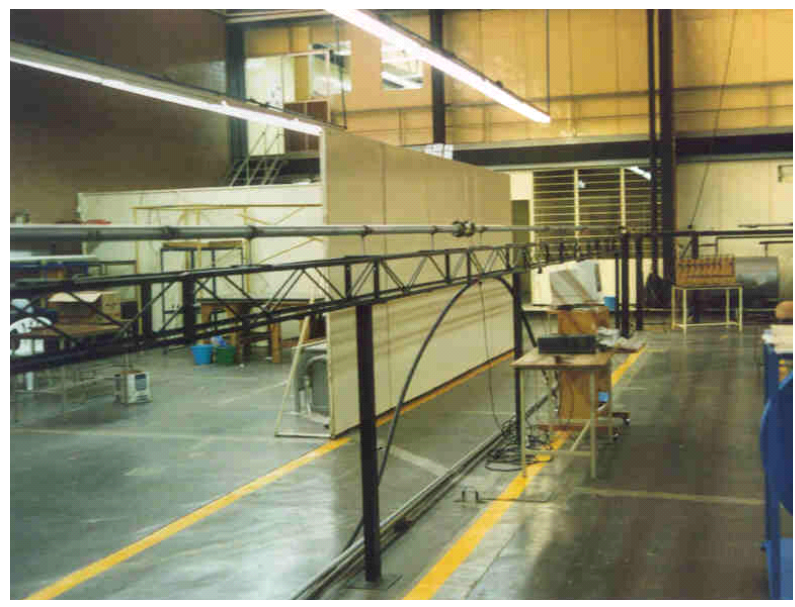

Figure 3. Panoramic view of the experimental loop.

Figure 5 presents the results of the 0.75 inches elbow. Here can be seen that $\Delta \mathrm{P}_{\mathrm{r}}(\mathrm{elbow})$ is greater than $\Delta \mathrm{P}_{\mathrm{p}}$ (orifice plate). The tendency for the other elbows is the same but we can say that $\Delta \mathrm{P}_{\mathrm{r}}$ grows as the curvature radius decreases and so, the flow rate calculation is more reliable. However, data about curvature radius influence in the radial pressure drop are limited to specific applications. 


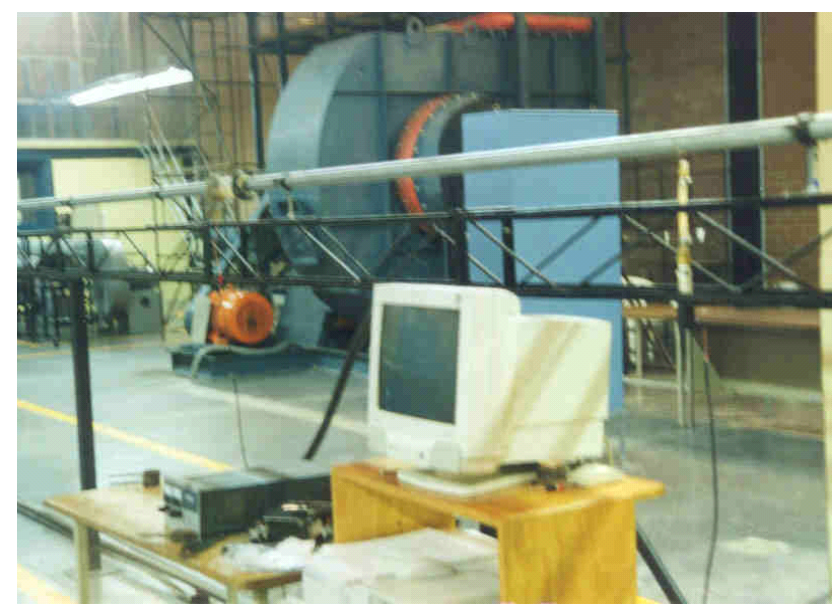

Figure 4. Details on work station.

Figure 6 shows the linear relationship between $\left(\Delta \mathrm{P}_{\mathrm{r}}\right)^{1 / 2}$ and the flow rate, both parameters are part of equation (4). The term:

$$
\frac{C_{c} p D^{2}}{4} \sqrt{\frac{1}{\rho \ln \left(D / r_{b}+1\right)}}
$$

is the slope of the straight lines in figure 6 and will be known as $\mathrm{K}$. K values for the straight lines were computed using the experimental data, and we obtained $0.056,0.03$ and 0.014 for the $1.5,1$ and 0.75 inches elbows, respectively. Parameter $\mathrm{K}$ varies with the diameter of the elbow, so it is possible to develop an expression in order to correlate the elbow diameter and K. Unfortunately, this correlation is not complete because the curvature radius has a great influence on $\Delta P_{r}$. If a $\frac{D}{r_{b}}$ relationship is defined, an expression for the $\mathrm{K}$ parameter can be deduced by linear regression. Figure 7 shows the comparison of the computed flow rate with the calibrated orifice plate and the flow rate computed with the elbow corrected equation.

$$
K=0.208 \frac{D}{r_{b}}-0.1023
$$

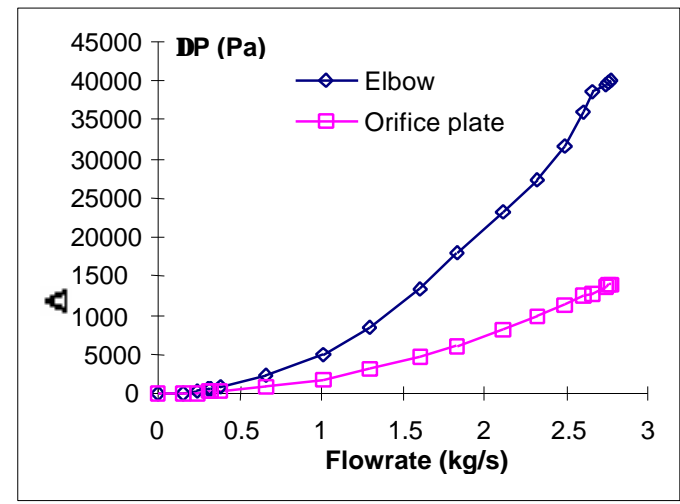

Figure 5 Experimental results for the 0.75 inches elbow 


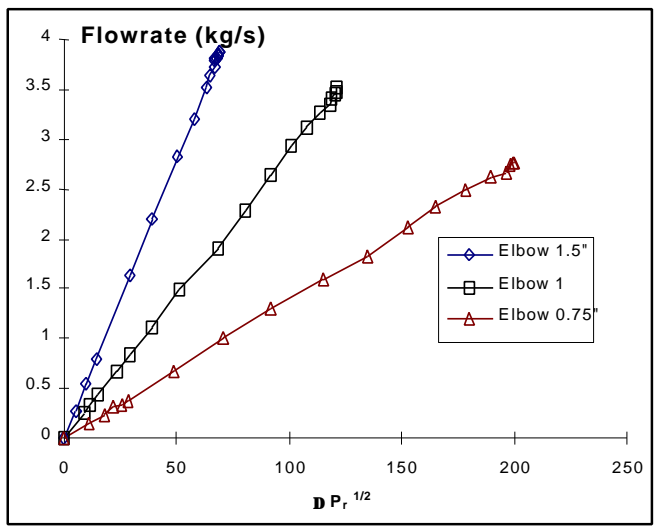

Figure 6 Linear Flowrate variation for all three elbows

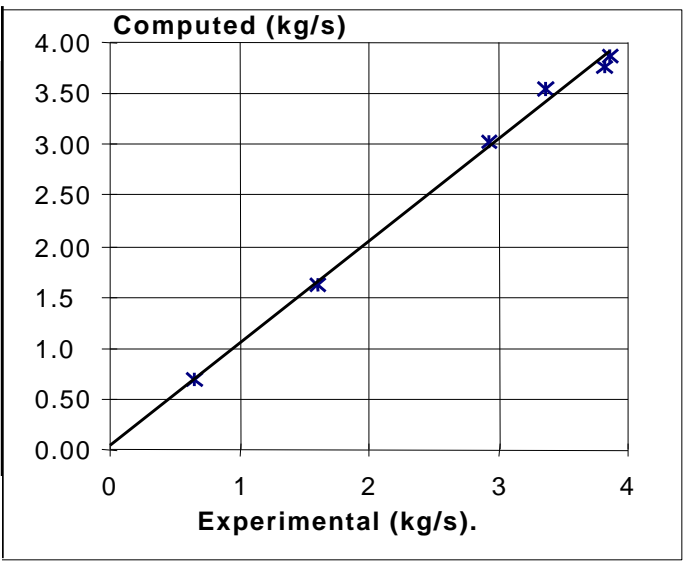

Figure 7 Experimental and computed flowrate

Other important parameter studied in the experimental work was the static pressure for different angular positions in the internal and external walls of the elbow. The curvature radius was $100 \mathrm{~mm}$. The pressure taps were located as shown in figure 8 , separated $15^{\circ}$ one from the other. The static pressure was measured using a calibrated pressure transducer; a $5 \mathrm{HP}$ centrifugal pump provided the water flow, which was measured with a calibrated orifice plate. The elbow had a far enough smooth pipe zone before and after the change of direction in order to avoid flow perturbations. The experimental flow conditions for each elbow are shown in table III.

Table III. Flow field conditions in control volume inlet.

\begin{tabular}{|c|c|c|}
\hline \multicolumn{3}{|c|}{ Fluid: water } \\
\hline Velocity, m/s & Reynolds Number & Flow rate, $\mathbf{k g} / \mathbf{s}$ \\
\hline 1 & 14637 & 1.52 \\
\hline 1.52 & 21956 & 2.28 \\
\hline
\end{tabular}




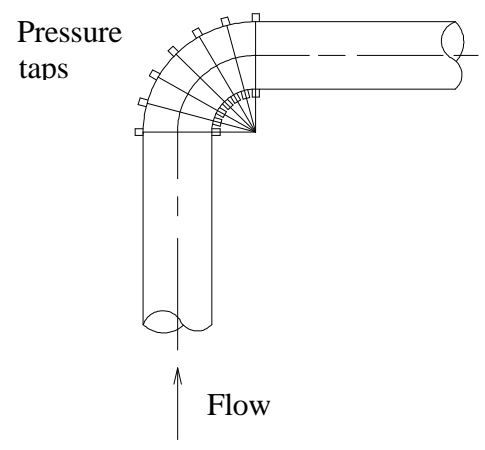

Figure 8. Experimental Zone

\section{NUMERICAL RESULTS}

Figures 9 and 10 show the pressure distribution in the elbow in a $X Y$ plane in color spectral. The plane coincides with the symmetry line of the control volume. It is clear that the internal wall, is the low-pressure zone, and the pressure grows following the direction of the external wall. In the $230-\mathrm{mm}$ radius curvature pipe, the pressure distribution has a different behavior than in the 20 and $100 \mathrm{~mm}$ due to the smaller effects of the centrifugal force. There are isobaric zones in the curved pipes; i.e. there is not differential pressure in these regions so, flow is zeroing, the fluid particles travel from the high-pressure zones to the low pressure ones, following elliptical trajectories. In both flow rates analyzed, the pressure difference behavior was similar. In these figures it can be observed that pressure grows in the external wall near to the inlet of the curvature. At $12^{\circ}$ approximately, pressure gets its maximum value. In the $230 \mathrm{~mm}$ curvature radius elbow, the pressure decreases in the axial direction and the pressure drops in a similar way than in a straight pipe, but the values in the internal and external wall are different.

Figures 11 and 12 show the radial pressure difference in the three elbows analyzed for the bulk velocity of $1 \mathrm{~m} / \mathrm{s}$ and $1.5 \mathrm{~m} / \mathrm{s}$ in the zone of developed flow respectively. It is clear that the maximum pressure difference decreases as the curvature radius grows, and it is also possible to observe that the point of the maximal difference goes to the left, that is, the position angle decreases. In both cases the pressure behavior is similar. There is a pressure difference due to the inlet and outlet curvature effects at $0^{\circ}$ and $90^{\circ}$ positions.

Since in the external wall there is a negative pressure gradient due to the centrifugal force, the boundary layer separation takes place before the $45^{\circ}$. Although in the internal wall there is a positive gradient at the inlet and due to the curvature, the boundary layer separation takes place after the $45^{\circ}$.

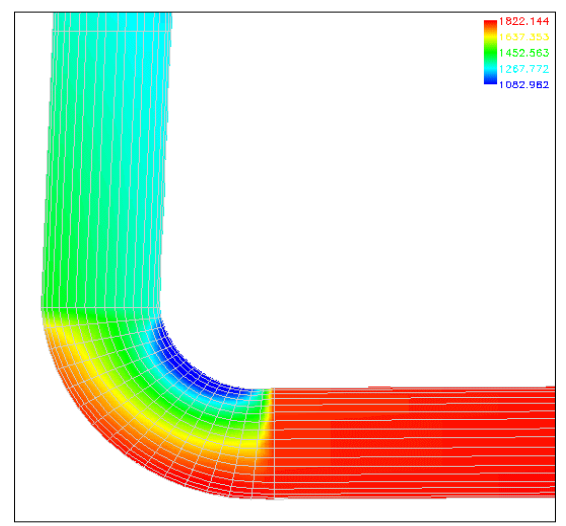

Figure 9. Pressure distribution in the 20-mm elbow. 
Figure 13 shows the velocity field in the $X$ direction for the flow in the curved pipe of 20 -mm curvature radius, the velocity is greater in the internal wall, and after the curvature, there is a small secondary flow in the $\mathrm{X}$ direction.

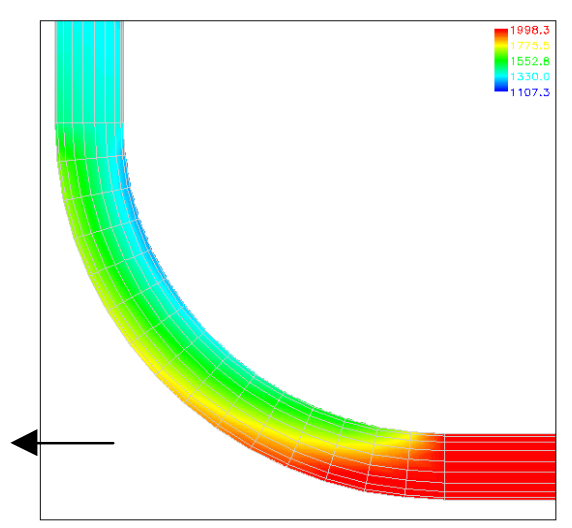

Figure 10. Pressure distribution in the 230-mm elbow.

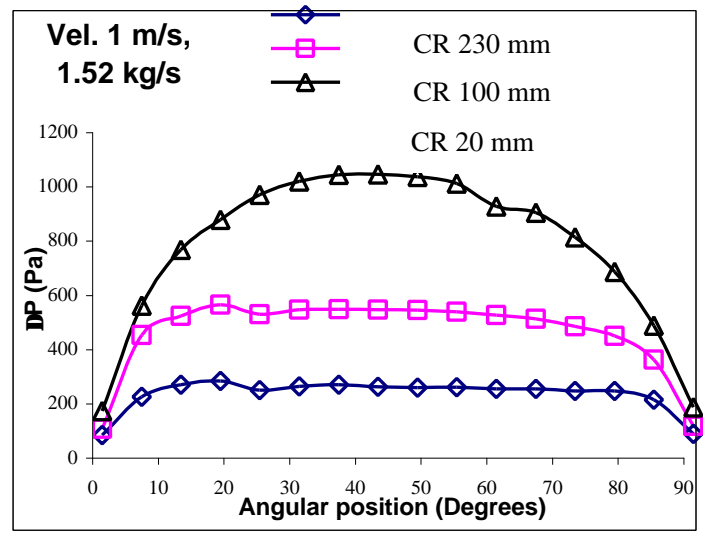

Figure 11. Radial pressure difference in the three elbows.

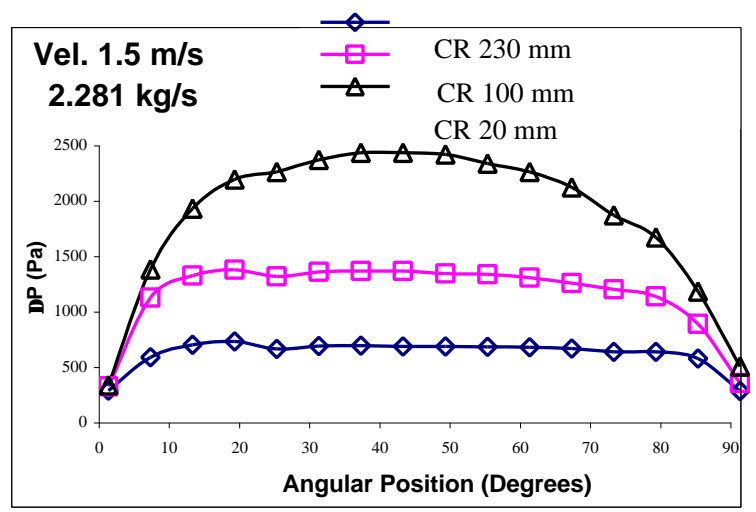

Figure 12. Radial pressure difference in the three elbows.

So the elliptical movement acquired in the curvature persists in the straight zone after the direction change. 


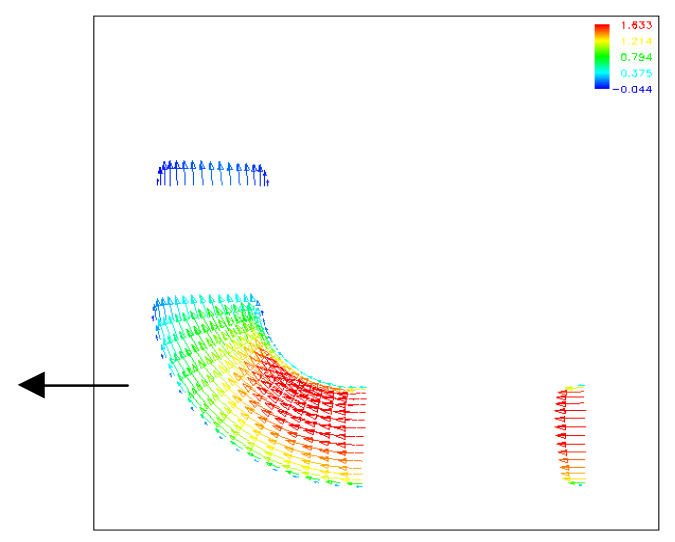

Figure 13. Velocity distribution $20 \mathrm{~mm}$ curvatura

\section{RESULTS COMPARISON}

Figure 14 shows the results for the 100-mm curvature radius. We can observe that pressure values in the internal and external wall of the elbow are not the same although their tendency is similar.

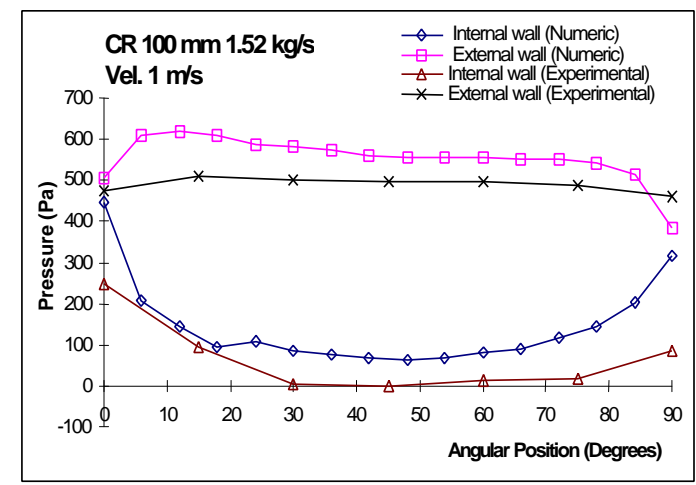

Figure 14. $100 \mathrm{~mm}$ curvature radius, $1.5 \mathrm{~kg} / \mathrm{s}$ flow rate.

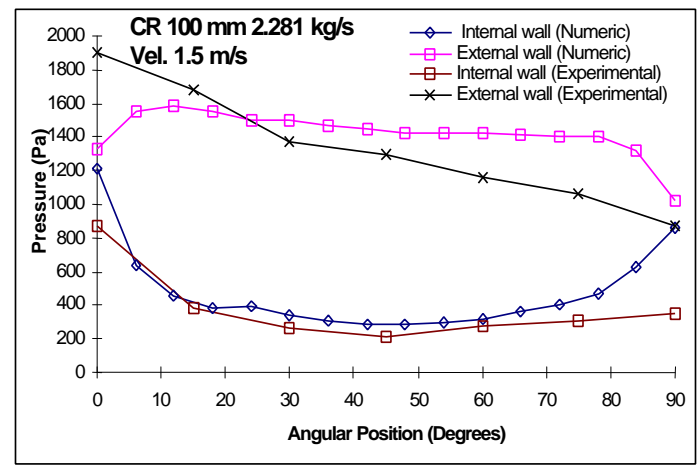

Figure 15. 100-mm curvature radius, $2.3 \mathrm{~kg} / \mathrm{s}$ flow rate.

The maximum radial pressure difference in both analyses is located between $15^{\circ}$ and $45^{\circ}$ and the pressure values in the internal wall for the $2.281 \mathrm{~kg} / \mathrm{s}$ flow rate (figure 15) have concordance in the numerical and 
experimental analysis but in the external wall the values are similar. It is important to remark that in experimental results there is an important inlet and outlet influence, but the tendency is similar in both cases.

\section{FLOW MEASUREMENT PRESSURE TAPS}

From the numerical and experimental results, the maximal radial pressure difference is located between $15^{\circ}$ and $45^{\circ}$. Since the objective of this work is to know, as a function of Reynolds number and curvature radius, where the maximal radial pressure difference occurs, a numerical relation can be obtained from the results shown in figures 11 and 12 . For the $20-\mathrm{mm}$ curvature radius the maximum radial pressure difference angle diminishes as the Reynolds number grows. For the 100 and $230 \mathrm{~mm}$ curvature radius this angle is constant and depends on the Reynolds number. Assuming a linear behavior for this angle, there is a horizontal line from the 100 and $230-\mathrm{mm}$ radius, whereas the line is inclined for the $20-\mathrm{mm}$ curvature radius. Since the difference between this characteristic lines are their slope and a constant "b", then, the lines for others curvatures could be assumed as straight lines too, but their slope is between the two references, covering different curvature radiuses. Then the equation for these lines could be express as:

$$
\theta_{\max }=62.99-0.45 r-0.001 R e+1 \times 10^{-5} r R e
$$

\section{CONCLUSIONS}

The results of this study show that there is a great viability for the use of elbows as flowmeters. It was observed that as the internal diameter of the elbow decreases the radial pressure difference increases and the accuracy for the flow rate computation grows. This behavior can be explained by equation (2), although this equation is ideal, it can be seen that the curvature radius is the main factor for the $\Delta \mathrm{P}_{\mathrm{r}}$.

After the numerical and experimental results we can conclude that the maximal radial pressure difference zones in a $90^{\circ}$-elbow are located between $15^{\circ}$ and $45^{\circ}$ depending on the Reynolds number and curvature radius. Since in every experimental work there are always measuring errors, the ones occurred in this work are due to the perpendicularity of the pressure taps and imperfections of the elbow, which provokes distortions in the flow field. It is important to know that the curvature ratio $d / R$ analyzed in this work were 2.2, 0.44 and 0.191 respectively for the numerical analysis. A bigger diameter would not have the same behavior because of the bigger transversal and longitudinal area of the flow, which would affect the boundary layer behavior, so it is necessary to study different diameters and flow conditions in order to have a better comprehension of this phenomena.

The best results are obtained with the small curvature elbows. Any way, there is always necesary to calibrate the elbow against a more accurate instrument.

\section{REFERENCES}

[1] British Standards Institution. BS 1042: Section 1.1: 1981. "Methods of measurement of Fluid Flow in closed conduits". Part 1. Pressure differential devices.

[2] Cheng, G. C.; Farokhi, S. "On turbulent flows dominated by curvature effects." Journal of Fluids Engineering. Vol. 114 March 1992, pp. 52-57.

[3] Dally, W.; Riley, W. F.; McConell, K. G. "Instrumentation for Engineering Measurements", John Wiley \& Sons, Inc., 1993, Second Edition.

[4] Ghia, K. N.; Ghia, U.; Shin, C. T. "Study of fully developed incompressible flow in curved ducts, using a multigrid technique." Journal of Fluid Engineering. Vol. 109. September 1987, pp. 226-236.

[5] Idelchik, E. Handbook of Hydraulic Resistance. 3rd. Edition. CRC Press, Inc. 1994.

[6] Launder, B. E.; Spalding, D. B. Mathematical models of Turbulence. Academic Press London. 1972. 
[7] Launder, B. E.; Spalding, D. B. Spalding. "The numerical computation of Turbulent Flows." Computer methods in Applied Mechanics and Engineering, Vol. 3, pp. 2269-289. 1974.

[8] Orlandi, P.; Cunsolo, D. "Two-dimensional laminar flow in elbows." Journal of Fluid Engineering. Vol. 101. June 1979, pp. 276-283.

[9] Rindt, Steenhoven, Janssen and Vossers. "Unsteady entrance flow in a $90^{\circ}$ curved tube." Journal of Fluid Mechanics. Vol. 226, 1991, pp. 445-474.

[10] Sudo, K. Sumida, M. Yamane, R. "Secondary motion of fully developed oscillatory flow in a curved pipe." Journal of Fluid Mechanics. Vol. 237. 1992, pp. 189-208.

[11] White, F. M. Viscous Fluid Flow. McGraw-Hill International Editions. Mechanical Engineering Series. 1991.

\section{NOMENCLATURE}

A cross sectional area

$C_{p} \quad$ total coefficient for orifice plate

$C_{c} \quad$ total coefficient for elbow

$d$ diameter of orifice plate

$D \quad$ internal elbow diameter

$K \quad$ slope of the straight lines in fig. 4

$Q \quad$ volumetric flow

Re Número de Reynolds

$r \quad$ elbow curvature radius

$r_{b} \quad$ internal elbow curvature radius

$r_{e} \quad$ external elbow curvature radius

$V \quad$ local velocity of the fluid

$\Delta P_{a} \quad$ axial pressure difference

$\Delta P_{p} \quad$ difference pressure in orifice plate

$\Delta P_{r} \quad$ radial difference pressure in elbow

\section{GREEKS}

$\kappa \quad$ turbulent kinetic energy

$\varepsilon \quad$ turbulence energy dissipation rate

$\rho$ density of the fluid

$v \quad$ Kinematic viscosity of the fluid 


\section{Authors Byography}

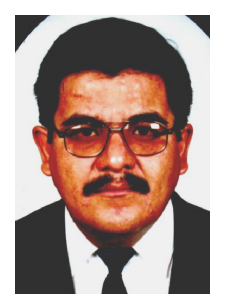

\section{ABDIEL GÓMEZ MERCADO}

Industrial engineer at Instituto de Ciencias Exactas de la Universidad Autónoma del Estado de Hidalgo (Exact Sciences Institute at Autonomous University of Hidalgo State, Mexico). He held a Masters degree in Mechanical Engineering at Escuela Superior de Ingeniería Mecánica y Eléctrica del Instituto Politécnico Nacional (Superior School of Mechanical and Electronic Engineering at National Polytechnic Institute). He was a researcher in two-phase flow and geothermal fluids area at Electrical Research Institute (IIE, 1988-1989) at Mexico City and Cuernavaca, Mor. Since 1989 he is a professor at Mechanical Engineering Department of the Instituto Tecnológico de Pachuca (Technological Institute Pachuca, Mexico) and currently is finishing his doctoral program in Mechanical Engineering at Graduate \& Research Department (SEPI) of ESIME-IPN. He has been working in two-phase flow dynamics research projects since 1998.

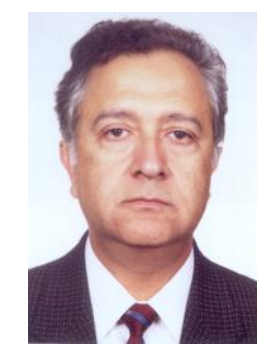

\section{PEDRO QUINTO DIEZ}

Mechanical engineer at Escuela Superior de Ingeniería Mecánica y Eléctrica of the Instituto Politécnico Nacional (Superior School of Mechanical and Electronic Engineering at National Polytechnic Institute). He held a PhD degree in Mechanical Engineering at National Institute of Applied Sciences, Lyon, France. Since 1975 he is a researcher and professor at Graduate \& Research Department (SEPI) of ESIME-IPN. Since 1979 he has been working in research projects in thermal energy and solar energy.

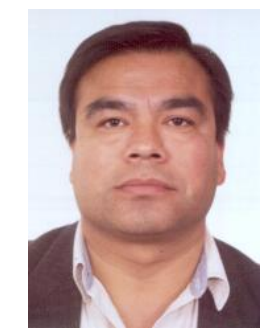

\section{FLORENCIO SÁNCHEZ SILVA}

Mechanical engineer at Escuela Superior de Ingeniería Mecánica y Eléctrica of the Instituto Politécnico Nacional (Superior School of Mechanical and Electronic Engineering at National Polytechnic Institute). He held a PhD degree in Mechanical Engineering at National School of Mechanics and Aero techniques, Poitiers, France. Since 1980 he has been working in research projects in twophase flow dynamics, heat transfer and energy save. He was a researcher in the area of geothermal fluids at Electrical Research Institute (IIE) at Mexico City (1980-1991).Since 1992 he is a researcher and professor at Graduate \& Research Department (SEPI) of ESIME-IPN and currently is chairman of this section. 